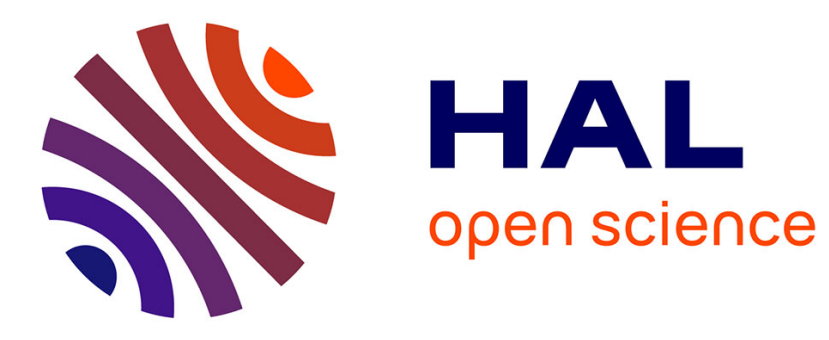

\title{
Handover management for hybrid satellite/terrestrial networks
}

Fabrice Arnal, Riadh Dhaou, Julien Fasson, Julien Bernard, Didier Barveaux, Emmanuel Dubois, Patrick Gelard

\section{- To cite this version:}

Fabrice Arnal, Riadh Dhaou, Julien Fasson, Julien Bernard, Didier Barveaux, et al.. Handover management for hybrid satellite/terrestrial networks. PSATS 2012 - 4th International Conference on Personal Satellite Services, Mar 2012, Bradford, United Kingdom. pp.9. hal-00750217

\section{HAL Id: hal-00750217 https://hal.science/hal-00750217}

Submitted on 9 Nov 2012

HAL is a multi-disciplinary open access archive for the deposit and dissemination of scientific research documents, whether they are published or not. The documents may come from teaching and research institutions in France or abroad, or from public or private research centers.
L'archive ouverte pluridisciplinaire HAL, est destinée au dépôt et à la diffusion de documents scientifiques de niveau recherche, publiés ou non, émanant des établissements d'enseignement et de recherche français ou étrangers, des laboratoires publics ou privés. 


\title{
Handover management for hybrid satellite/terrestrial networks
}

\author{
F. Arnal, R. Dhaou, J. Fasson, J. Bernard, D. Barvaux, E. Dubois and P. Gélard
}

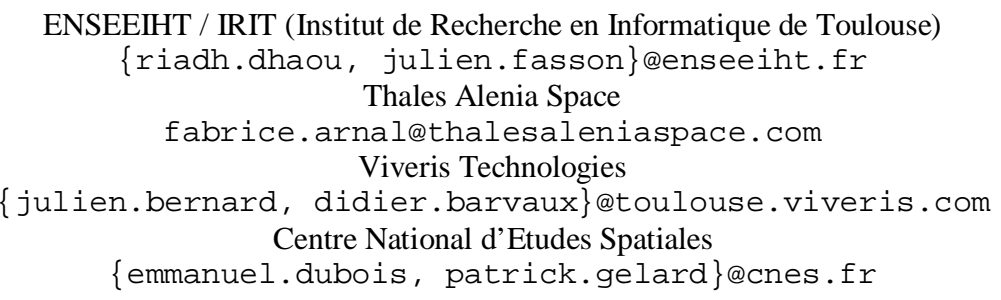

\begin{abstract}
Initially envisaged to support handover between different wireless 802.x network technologies, the IEEE 802.21 standard also appears as the good candidate for handover management in future integrated satellite / terrestrial systems. This paper presents an analysis of how this standard could be implemented in the frame of a realistic scenario and taking into account the current trends in wireless network and mobility architectures. Our solution is then evaluated by means of emulation over a DVB-RCS representative testbed, and based on an experimental MIH implementation. We finally show that seamless handover can nearly be achieved with very short service outages.
\end{abstract}

Keywords: MIH, Satellite, 3GPP, LTE, Handover, Mobility, Test-bed.

\section{Introduction}

These last years show a tremendous evolution of GEO systems towards the integration of terminal mobility. This association with wireless terrestrial networks is a relatively new idea. Several proprietary hybrid systems integrating GEO satellites and ground components arise. The design of these integrated/hybrid systems takes into account physical, MAC, and network layers issues [1]. The combination of these two technologies can achieve for broadband services full coverage and high capacity. However the convergence at network level is still an open issue.

The main networking trial is certainly seamless handover. Managing handover in a couple of heterogeneous technologies may rely either on specific optimised or on standard and generic solution such as Media Independent Handover (MIH). The first solution may be optimised for each case of technologies. The last solution evolves more naturally and easily in order to integrate new access technologies. However there is a clear lack of experience in the deployment of this kind of solution. Therefore, our position here is to propose realistic hybrid network architecture and to see how MIH could be integrated.

The paper is organized as follow: Section 2 presents existing works related to mobility and MIH backgrounds. Section 3 identifies a possible use case for satellite/terrestrial integration and describes a relevant network architecture and 
design choices for the MIH usage. At last, Section 4 presents the evaluation of this solution through experimentations lead on an emulation platform.

\section{Inter-system mobility architectures and MIH backgrounds}

The major standard organisations support mobility features:

- IETF proposes network, transport and session level mobility solutions, therefore usable for inter-system handover. The MIPSHOP and MEXT working groups focus on mobility issues in heterogeneous networks. Several mobile IP based solutions and extensions were proposed and a big trend has been raised on the development of "Smart Routers" products that are based on such solutions. Transport level mobility solutions are based either on TCP (MP-TCP, FreezeTCP and Mobile Socket Service) or on SCTP. SIP-based mobility was also proposed.

- 3GPP/3GPP2 architecture not only supports inter-technology mobility but also vertical handover (e.g. with WiFi and Wimax). Two operation modes are defined: the non-optimized and the optimized handovers. For instance, VoIP services, in a $2 \mathrm{G} / 3 \mathrm{G}$ network, are based on the first mode whereas no standardized solutions exist for the second one. The network level mobility is based on MIPv4, DSMIPv6 and PMIP (Proxy MIP).

- From another hand, IEEE has formed the 802.21 group, where MIH was formerly proposed for handover between 802.x access technologies. Later, the standard has been extended for $3 \mathrm{GPP}$ networks support.

MIH provides abstract services to the higher layers using a unified interface (L2.5 functionalities). MIH is not designed to take handover decision but rather provides the communication tools to assist the handover process. MIH defines three different services, i.e. Media Independent Event Service (MIES), Media Independent Command Service (MICS) and Media Independent Information Service (MIIS). MIES provides events triggered by changes in the link characteristic and status. MICS provides the upper layers necessary commands to manage and control the link behaviour to accomplish handover functions. MIIS provides information about the neighbouring networks and their capabilities. Extensive details on the IEEE 802.21 standard can be found in [2].

One major question is certainly the reason to use MIH in satellite context. Indeed, the needs of vertical handover schemes [3] have been shown in several architectures based on the 802.21 standard $[4,5,6]$.

\section{Existing studies on MIH, implementations and testbeds}

To our knowledge, there is no reference implementation of the IEEE 802.21 standard. Nevertheless, FP6/FP7 IST projects have contributed to a prototype on GNU/Linux [7]. Another prototype proposes an implementation of a mobile VoIP using SCTP with automatic address reconfiguration [8]. An experimental and analytical study was conducted [9] to determine the adequacy of protocols for mobility networks. This study presents the handover procedures (based on MIPv6 and 
PMIPv6) in WIFI/CDMA networks. PMIPv6 based on reactive handover method has been also observed in [10]. In [11], a 802.21 client is simulated in a heterogeneous environment and the paper analyzed the effect of speed on terminal handovers between WiFi and 3G, but without validation of these simulations. Last, MIH has been proposed for future aeronautical communications [12].

The use of MIH was also discussed in several prototypes. An implementation of 802.21 with a limited number of features was presented in [13]. In [14] the integration of broadcast technology in heterogeneous networks using MIH is discussed. [15] presents a solution for handover between heterogeneous networks that integrates MIH and Mobile Proxy Agent by defining an API performing the MIH Service Access Point. Eventually there is an extended research activity on seamless handover. Models of MIH are integrated to simulation tools such as Qualnet [16] and ns-2 [17]. Open source implementations such as openMIH [18] and Odtone [19] have been also set up.

\section{Scenario description}

\section{Mobile Services}

A hybrid system aims at offering access to users' services wherever they are by enlarging the system coverage. However, such systems only make sense if they offer a mobile service. For a single user to a whole group of users in the same vehicle, applications are miscellaneous, from voice applications to Internet access.

\section{Access networks}

Between the different types of satellites, GEO systems offer the best opportunity to cope with the terrestrial part in terms of coverage and cost. Apart private solutions, the main standards proposing mobility for GEO are GMR, the mobile version of DVB Return Channel for satellites (DVB-RCS+M) and DVB for Satellite Handheld (DVB$\mathrm{SH})$ that focus on mobile broadcast services. GMR technology has been designed for extension of the cellular world to satellite and thus it focuses on voice services. Some integrated systems, like Terrestar [20] are nowadays using GMR technology to interconnect it to a mobile operator core network. Indeed GMR standard has evolved, following the "all-IP" wave. Therefore a LTE solution adapted to satellite appears as the right direction for GMR standard. In Ku/Ka bands, the use of DVB-RCS+M is highly relevant for vehicular services (i.e. mobile collective terminal) and this will be retained for our reference scenario, including for the test-bed implementation.

Concerning the terrestrial networks, we could consider any kind of terrestrial networks that may provide mobility. If LTE and Wimax are two promising solution, the limits of test-bed have induced the use of a simpler technology, WIFI.

\section{Network architecture}

A major element in network architecture is the different roles undertaken. We assume a mobile service provider (MSP) ensures the IP connectivity of its mobile clients (final users and/or hybrid terminal) and may provide some other kinds of services. This role is generally linked to the core network of final user. MSP is in charge of 
interaction with the manager of the different access networks that its clients are using. If needed, it may rent bandwidth at user's demand. The following Fig. 1 illustrates a distribution of roles through a DVB-RCS+M / LTE architecture. A group of users are connecting through a LAN in a train to their services. The hybrid terminal can connect to an E-nodeB or to the satellite Gateway. Finally, a Mobile IP / NEMO architecture is chosen for network mobility support.

The terrestrial and satellite networks are access networks between the user and his MSP. The MSP may propose the first visible router to user and it manages the AAA functionalities. However, some of these functionalities can be delegated to access network, using MSP Point of Presence (PoP). Moreover, MSP role can directly be undergone by one of the two access networks, especially for the 3GPP one. This option is more convenient for LTE proposing a whole set of services like IP Multimedia Subsystem (IMS) as shown on the figure. For satellite systems or Wimax the core network is less developed than cellular one. So this integration demands more change to their network architectures.

Policing functionalities can be distributing on both sides and centralized by a global AAA entity in the MSP. In the case of a LTE network this function is managed by the Policy and Charging Resource Function (PCRF) whereas for others access networks the control at IP level can be made in implementing the IETF COPS standard. Policy Enforcement Point (PEP) and Policy Decision Point (PDP) are then introduced in the architecture, the former being deployed in the access network while the later is directly managed by the Mobile Service Provider (MSP). The interaction between these functionalities and the handover signaling is however out of the scope of this paper.

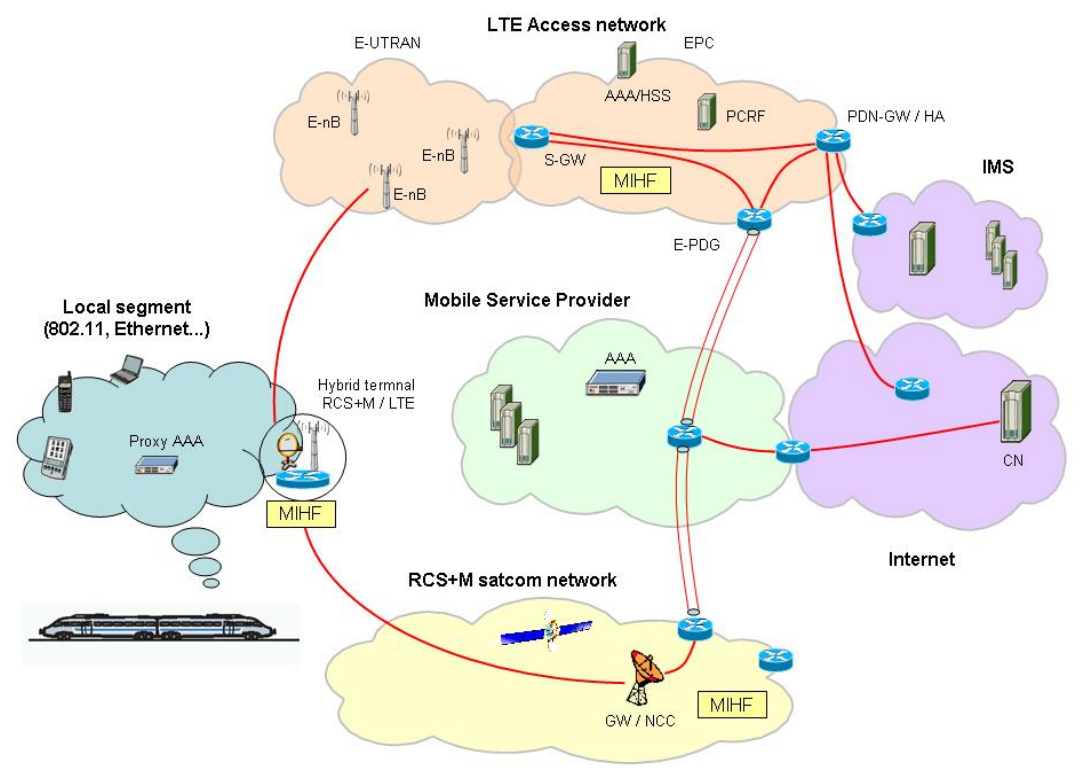

Fig. 1. An example of network architecture for the DVB-RCS+M/LTE reference scenario 


\section{Handover decision}

MIH supports both mobile-initiated handover as well as network-initiated handover, both alternatives can be preferred according to the exact deployment. In our scenario, the terminal chooses its mode of connection and the timing to perform the handover. A simple decision algorithm is implemented as an independent process (as a MIH user) in the terminal and based on predefined Signal-To-Noise Ratio thresholds. Thanks to this modular architecture, the handover decision and/or policies is open and could be easily extended with much sophisticated algorithms.

\section{MIH functionalities}

The MIH Function (MIHF) aims at referencing any event and at informing its users. In our proposal, MIHF is present in mobile and network elements, as it is the most general solution. For Wimax, MIHF is integrated on the access points whereas for LTE it may be the PDN-GW. However if the MSP takes part in the handover decision, it must have an MIHF so as to communicate with the handover decision function. MIHF is communicating with other MIHF in the different networks and they collect information, inform or receive order from the MIH users on the same entities. Another interesting MIH user is the MIIS server which can be used to select the available networks. However this option has not been chosen here.

\section{MIH primitive selection}

Many primitives are defined in the MIH standard although all are not necessarily required to be implemented. The selection of the right primitives may allow a seamless handover. The selection has been lead by the need to obtain a seamless handover and the convenience of the deployment for the tests. The following primitives have been used for handover indication:

- MIH_Link_Configure_Thresholds is used to fix the thresholds for signaling the need of a handover before the link is going done.

- MIH_Link_Parameters_Report proposes to inform the different entities when the threshold is reached. It allows the preparation of the handover.

- $\quad$ MIH_Link_Going_Down is used by the L2 interface to announce the near link outage.

\section{Implementation}

\section{Description of the emulation test-bed, implementation and scenario}

The core platform used is the Platine emulation tool [21], representative of a DVBS2/RCS access network and running under a Linux environment. NEMO was integrated to Platine from the UMIP v0.4 software upgraded with the NEPL patch [22]. Standard Linux Route Advertisements (RADVD) are used for address autoconfiguration. Finally the ODTONE v0.2 beta stack was used for MIH implementation, modified by minor adaptations for our needs. In order to model the LTE/3GPP connection we have used a WIFI link which was more convenient for performing tests in our lab environment. In addition, only the handover from WIFI to 
satellite could have been tested, as few extra developments were further needed for Satellite to WIFI handover in our platform.

The (emulated) mobile network is connected through a hybrid Satellite Terminal that can be connected to the satellite gateway through the emulated satellite link, or to the WIFI access point. The NEMO instance and the MIH local function are therefore deployed on the Satellite Terminal, while the satellite and WIFI gateways host the network-side MIH Functions.

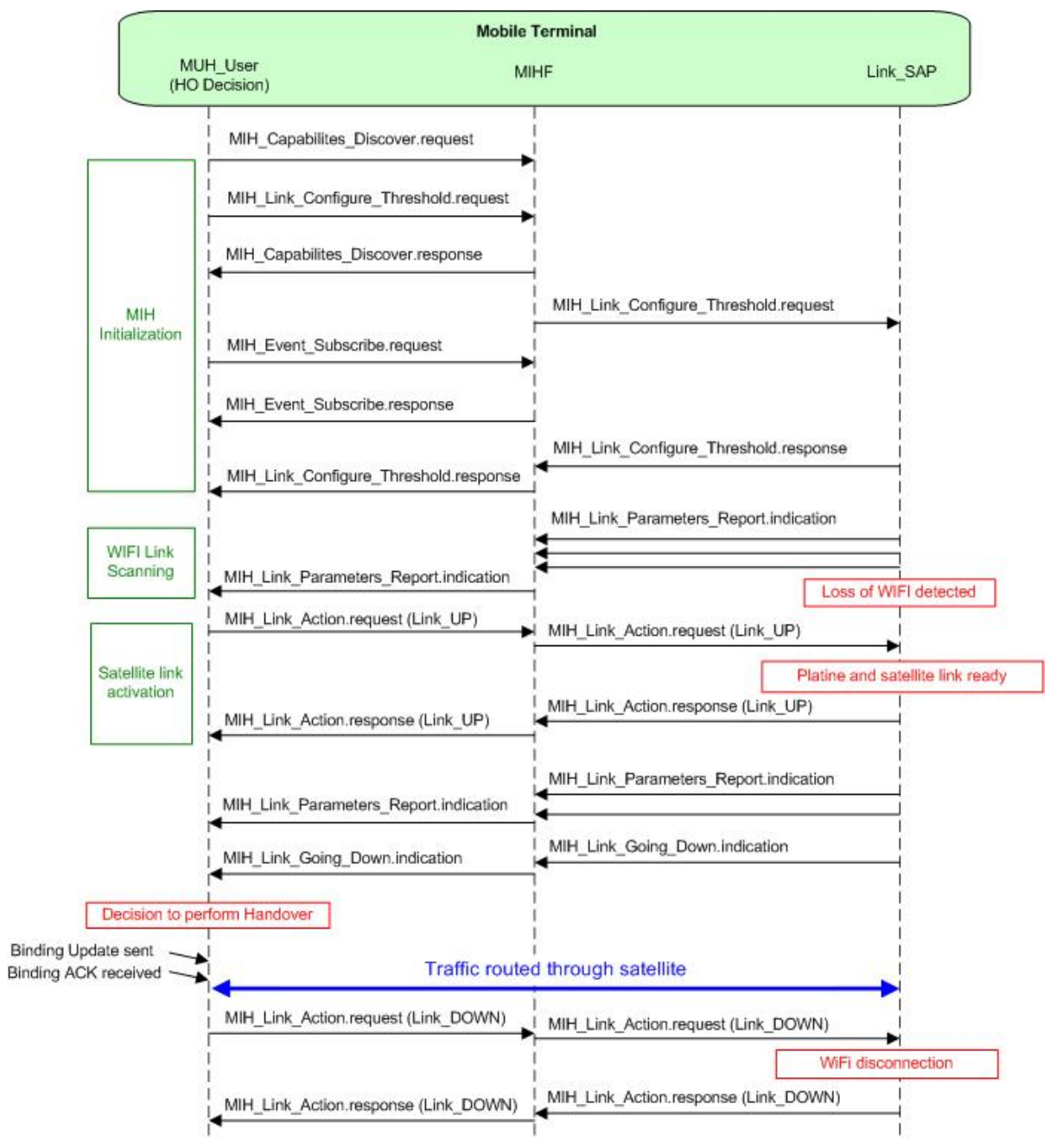

Fig. 2. MIH sequence diagram between the MIH user, MIH functionality and test-bed

Signal-to-Noise Ratio (SNR) variations are emulated by means of a pre-computed scenario stored in a file indicating the virtual SNR measurements. They are polled each 5 seconds by the mobile MIH Function (LINK_SAP interface). Two SNR thresholds are configured at the MIH stack (at the MIH startup, with 
LINK_CONFIGURE_THRESHOLD). Each threshold is mapped to a given MIH event (LINK_GOING_DOWN and LINK_PARAMETER_REPORT). The first event, when received, is used to start the Platine logging process. If successful, Route Advertisements can be received for the satellite connection through the virtual Platine network interface. Subsequently, when the second threshold is crossed, it triggers the NEMO handover phase. We capture this event to send an inter-process signal (SIGUSR2) to the NEMO stack in order it performs the Binding Update / Acknowledgement procedure. For future improvements, this interworking will be developed in a proper module that will also implement separately the Decision algorithm for flexible evolutions.

Application flows are composed of a CBR audio and a VBR video stream (RTP/UDP), each generated by VLC at a host located in the mobile network, and sent to a Correspondent Node $(\mathrm{CN})$. This receiver is located at a neighboring network interconnected to both WIFI and satellite networks.

\section{Results}

A Wireshark client running at the $\mathrm{CN}$ measures the incoming rates. As shown in Fig. 3 , a short outage ( 0.6 seconds) is experienced just after the handover at second 90 . At the receiver, the audio flow starts to be corrupted, but the video quality remains intact.

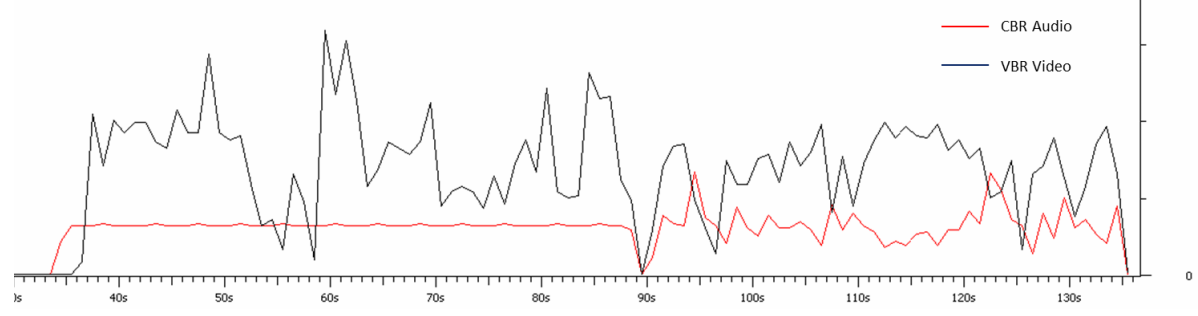

Fig. 3. Incoming bit rates variations measured at Correspondent Node

Our investigation shows that no packet loss happens during the handover, which is a first point of satisfaction. With comparison to a basic scenario test conducted in the same conditions but without MIH, that cannot be presented here due the lack of space, a packet loss sequence lasting nearly for 4.5 seconds was observed.

Immediately after the handover, we understood in observing an increase of the satellite queue utilization that the introduced delay is not related to any mobility latency but to the satellite access layer allocations loop (Rate-Based Dynamic Capacity allocations were used here). In addition, the sudden propagation delay change (tenths of milliseconds with WIFI, 300ms with satellite) also adds a contribution to the observed delay. However since the video client largely buffers the receiving flow, this latency effect remains transparent, whereas audio samples are by far less buffered.

For the remaining period, audio remains corrupted and not video. This is due to the absence of any QoS support on the overloaded satellite connection. Buffering at the satellite link layer continues is never completely absorbed. Except this effect, we 
conclude this MIH integration is successful and achieves good performances, though only based on an experimental stack.

\section{Conclusion}

This paper has demonstrated that $\mathrm{MIH}$, that appear complex at first glance, is actually well applicable to any hybrid IP satellite network, such as the future LightSquared system in the US based on LTE. Coupled with Mobile IP network mobility, it is able to show very good performances provided that the loss of signal can be detected before the link completely goes down. In our future works, derived scenarios with the support of QoS will be presented in an IMS environment, in order to illustrate how resources could be provisioned before the mobile switches to another network. We also intend to complete our integration work by supporting Satellite to WiFi handover, and maybe to implement handover decisions based on real SNR and/or location measurements.

\section{References}

1. S. Kota, G. Giambene and S. Kim, "Satellite component of NGN: Integrated and hybrid networks", Int. J. of Satellite Communications and Networking, May 2010, Wiley.

2. IEEE 802.21 "IEEE Standard for Local and metropolitan area networks- Part 21: Media Independent Handover Services", 2009

3. J. Márquez-Barja et al., "An overview of vertical handover techniques: Algorithms, protocols and tools", Computer Communications 34 (2011) 985-997.

4. M. Salhani, R. Dhaou, A.-L. Beylot, "Terrestrial Wireless Networks and Satellite Systems Convergence", Int. Communications Satellite Systems Conference, AIAA, 2007.

5. R. Qureshi,A. Dadej, "Adding Support for Satellite Interfaces to 802.21 Media Independent Handover", ICON 2007.

6. Y.F. Hu et al., "Mobility Extension for Broadband Satellite Multimedia", IWSSC 2009.

7. Piri, E. \& Pentikousis, K., “Towards a GNU/Linux IEEE 802.21 Implementation”, Proc. IEEE International Conference on Communications ICC '09 , pp. 1-5, 2009

8. Chen, Y-M. et al., "SCTP-based handoff based on MIH triggers information in campus networks", $8^{\text {th }}$ International Conference Advanced Communication Technology ICACT 2006, Vol. 2, pp. 5pp.-1301, 2006

9. T. Chiba, H. Yokota, A. Dutta, D. Chee, H. Schulzrinne, "Performance Analysis of Next Generation Mobility Protocols for IMS/MMD Networks", Proc. International Wireless Communications and Mobile Computing Conference IWCMC '08, 2008, pp. 68-73

10. Choi, H.Y., Min, S.G., Kim, K.R., Han, Y.-H. \& Lee, H.B. , "Seamless handover scheme for proxy mobile IPv6 using smart buffering", International Conference on Mobile Technology, Applications, and Systems, ACM, 2008, pp. 1-7

11. T. Melia et al., "Analysis of effect of mobile terminal speed on WLAN/3G vertical handovers", IEEE Globecom 2006.

12. S. Ayaz et al., "Architecture of an IP-based Aeronautical Network to Integrate Satellite and Terrestrial Data Links", ICNS, May 2009

13. A. Dutta et al., "Seamless Handover across Heterogeneous Networks - An IEEE802.21 Centric Approach,” IEEE WPMC, 2006. 
14. T. Buburuzan et al., "Integration of Broadcast Technologies with Heterogeneous Networks - An IEEE 802.21 Centric Approach”, Consumer Electronics, ICCE 2007

15. M. Tauil et al., "Realization of IEEE 802.21 services and pre-authentication framework", Int. Conference on Testbeds and Research Infrastructures for the Development of Networks. Communities and Workshops TridentCom 09, pp. 1-10, 2009

16. P. Latkoski et al., "SDL+QualNet: A Novel Simulation Environment for Wireless Heterogeneous Networks", SIMUTools 2010, Torremolinos, Malaga, Spain, March 2010.

17. R. Rouil, N. Golmie, N. Montavont , "MIH transport using cross-layer optimized stream control transmission protocol", Computer Communications 33 (2010) 1075-1085.

18. Y. Lopez and E. Robert, "OpenMIH, an Open-Source MIH Implementation and Its Application to Proactive pre-Authentication", K. Pentikousis et al. (Eds.): MONAMI 2009, LNICST 32, pp. 14-25, 2010.

19. D. Corujo et al., "Using an open-source IEEE 802.21 implementation for network-based localized mobility management", IEEE Communications Magazine, Volume 49, Issue 9, see http://helios.av.it.pt/projects/odtone

20. B.Vojcic, D. Mathesson, H. Clark, "Network of Mobile Networks; Hybrid TerrestrialSatellite Radio", IWSSC 2009

21. C. Baudoin, F. Arnal, "Overview of Platine emulation testbed and its utilization to support DVB-RCS/S2 evolutions", ASMS 2010, Cagliari, Italy, 13-15 Sept. 2010

22. http://software.nautilus6.org/NEPL-UMIP/index.php 